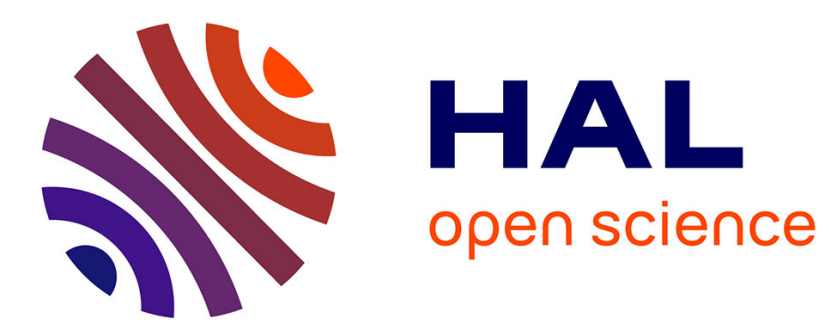

\title{
The concept of Cyclic Sound Intensity and its application to acoustical imaging
}

B. Lafon, Jérôme Antoni, Ménad Sidahmed, Laurent Polac

\section{To cite this version:}

B. Lafon, Jérôme Antoni, Ménad Sidahmed, Laurent Polac. The concept of Cyclic Sound Intensity and its application to acoustical imaging. Journal of Sound and Vibration, 2011, 330 (9), pp.2107-2121. 10.1016/j.jsv.2010.11.003 . hal-01018741

\section{HAL Id: hal-01018741 \\ https://hal.science/hal-01018741}

Submitted on 3 Sep 2021

HAL is a multi-disciplinary open access archive for the deposit and dissemination of scientific research documents, whether they are published or not. The documents may come from teaching and research institutions in France or abroad, or from public or private research centers.
L'archive ouverte pluridisciplinaire HAL, est destinée au dépôt et à la diffusion de documents scientifiques de niveau recherche, publiés ou non, émanant des établissements d'enseignement et de recherche français ou étrangers, des laboratoires publics ou privés. 


\title{
The concept of cyclic sound intensity and its application to acoustical imaging
}

\author{
B. Lafon ${ }^{\mathrm{a}, *}$, J. Antoni ${ }^{\mathrm{a}, *}$, M. Sidahmed ${ }^{\mathrm{a}}$, L. Polac ${ }^{\mathrm{b}}$ \\ a University of Technology of Compiègne (UTC), Laboratory Roberval of Mechanics, Centre of Research of Royallieu, rue Personne de Roberval, \\ 60200 Compiègne, France \\ ${ }^{\mathrm{b}}$ RENAULT, Centre Technique de Rueil, CTR A03 3 37, 67 rue des bons raisins, 92500 Rueil Malmaison, France
}

\section{A R T I C L E I N F O}

\section{Article history:}

Received 12 October 2009

Received in revised form

17 September 2010

Accepted 3 November 2010

Handling Editor: K. Shin

Available online 10 December 2010

\begin{abstract}
A B S T R A C T
This paper demonstrates how to take advantage of the cyclostationarity property of engine signals to define a new acoustical quantity, the cyclic sound intensity, which displays the instantaneous flux of acoustical energy in the angle-frequency domain during an average engine cycle. This quantity is attractive in that it possesses the ability of being instantaneous and averaged at the same time, thus reconciling two conflicting properties into a rigourous and unambiguous framework. Cyclic sound intensity is a rich concept with several original ramifications. Among other things, it returns a unique decomposition into instantaneous active and reactive parts. Associated to acoustical imaging techniques, it allows the construction of sound radiation movies that evolve within the engine cycle and whose each frame is a sound intensity map calculated at a specific time - or crankshaft angle - in the engine cycle. This enables the accurate localisation of sources in space, in frequency and in time (crankshaft angle). Furthermore, associated to cyclic Wiener filtering, this methodology makes it possible to decompose the overall radiated sound into several noise source contributions whose cyclic sound intensities can then be analysed independently.
\end{abstract}

(c) 2010 Elsevier Ltd. All rights reserved.

\section{Introduction}

The engine encompasses the main sources of noise in a car. The radiated noise results from a mixture of various complex sources such as combustion, injection, mechanical shocks, etc. whose reduction is an important goal of the car industry. In that respect, specific techniques such as acoustic imaging and noise source separation that can assist the engineers in localising, identifying and ranking the main sources of noise are of prime concern. The aim of this paper is to demonstrate how the capabilities of these techniques can be notably enlarged, and their performance improved, by taking advantage of a particular statistical property of engine signals, referred to as cyclostationarity.

A cyclostationary signal is one whose statistical properties are evolving periodically with time $[1,2]$. When defined on the first order it means the signal contains some periodic components; on the second order it means the signal may be random while its energy is flowing periodically with time. It has been recently shown that engine signals - e.g. sound pressure or vibrations - are cyclostationary on the first and second order under steady operating conditions [3,4]. This property was then found extremely fruitful to define new processing tools dedicated to sound and vibration analyses of engines [5-7], just as it did in related areas concerned with the monitoring of rotating machines [8-12] or, with less obvious connections, in

\footnotetext{
* Corresponding authors.

E-mail addresses: brice.lafon@tremplin-utc.net (B. Lafon), jerome.antoni@utc.fr (J. Antoni).
} 
communications [13]. To the authors' knowledge and as far as acoustical imaging of engines is of concern, the cyclostationary property had never been exploited apart from very recently in [14,15].

One of the original results of the present study is the exploitation of cyclostationarity to define a new acoustical quantity, the cyclic sound intensity, which reflects the instantaneous flux of energy radiated per unit of time during an "average" engine cycle. This quantity is attractive in that it possesses the ability of being "instantaneous" and "averaged" at the same time, two conflicting properties which are reconciled here in a rigourous and quite unique framework. The so-defined cyclic sound intensity then serves as the backbone of the paper to support the introduction of other dedicated techniques. First, cyclostationarity is exploited to perform noise source separation by means of the cyclic Wiener filter [16-18], the originality of the approach lying in the post-processing of the separation results with the cyclic sound intensity. This enables a decomposition of the overall sound radiation into its contributions stemming from specific sources, thus assisting the user in understanding its physical origins and its propagation paths. Second, the cyclic sound intensity field is displayed as a sound radiation movie, whose each frame corresponds to a sound intensity map at a given time - or crankshaft angle - within the engine cycle, thus allowing a very precise localisation of sources in space, frequency, and time.

The paper is organised as follows. The first part is concerned with a brief reminder of the property of cyclostationary signals. In the second part, the concept of cyclic sound intensity is introduced with its specificities when devoted to engine signals. The third part then deals with the applications of cyclic sound intensity in noise source separation by means of the cyclic Wiener filter and in the construction of movies for visualising how sound instantaneously radiates.

\section{Theory of cyclostationarity}

This section briefly reviews the cyclostationary property that engine signals happen to enjoy. A formal definition is first provided in terms of the expectation operator, which has the advantage of conciseness. The correspondence with the timesynchronous average, the only operation that can be performed in practice, is then drawn through the concept of cycloergodicity.

\subsection{Definitions}

A stochastic process $x(t)$ is said to be cyclostationary of order $i$ and of period $T$ if any of its $i$-th order statistics is a periodic function of time with main period $T$. In the present work, cyclostationary processes of orders 1 and 2 - the so-called widesense cyclostationarity - will be considered only. Namely, $x(t)$ is said to be cyclostationary of order 1 and of period $T$ if its firstorder moment, as returned by the expected value $m_{x}(t) \hat{=} \mathbb{E}\{x(t)\}$, exists and is periodic of period $T$, viz.:

$$
m_{x}(t)=m_{x}(t+T)
$$

Similarly, $x(t)$ is said to be cyclostationary of order 2 if its autocorrelation function $R_{x x}(t, \tau) \hat{=} \mathbb{E}\{x(t) x(t-\tau)\}$ exists and is periodic of period $T$ :

$$
R_{x x}(t, \tau)=R_{x x}(t+T, \tau) .
$$

Eventually, two stochastic processes $x(t)$ and $y(t)$ are said to be jointly cyclostationary of period $T$ if their cross-correlation function $R_{y x}(t, \tau) \hat{=} \mathbb{E}\{y(t) x(t-\tau)\}$ is $T$-periodic:

$$
R_{y x}(t, \tau)=R_{y x}(t+T, \tau)
$$

\subsection{Cycloergodicity and time-synchronous averaging}

Closely linked to the property of cyclostationarity is the concept of cycloergodicity. A stochastic process $x(t)$ is said to be cycloergodic of order 1 and of period $T$ if its time-synchronous average tends to a certain (i.e. deterministic) function of time:

$$
\langle x(t)\rangle_{T} \hat{=} \lim _{K \rightarrow \infty} \frac{1}{2 K+1} \sum_{k=-K}^{K} x(t+k T)=\mu_{x}(t) .
$$

Therefore, if $x(t)$ is first-order cyclostationary of period $T$, then $\mathbb{E}\{x(t)\}$ is periodic by definition and clearly

$$
\mathbb{E}\{x(t)\}=\langle\mathbb{E}\{x(t)\}\rangle_{T} .
$$

Moreover, if $x(t)$ is also cycloergodic of the same period $T$, then by definition:

$$
\langle x(t)\rangle_{T}=\mathbb{E}\left\{\langle x(t)\rangle_{T}\right\} .
$$

Assuming that the operators $\mathbb{E}\{\cdot\}$ and $\langle\cdot\rangle_{T}$ commute, these last two equations imply the equality,

$$
\mathbb{E}\{x(t)\}=\langle x(t)\rangle_{T},
$$

meaning that for a first-order cyclostationary and cycloergodic process the expected value is equivalent to time-synchronous averaging. This property will be of fundamental importance in practice since it guaranties that the first-order moment $m_{x}(t)$ involved in Eq. (1) can be approached arbitrarily close by averaging enough cycles. Obviously, the same concept of timesynchronous averaging can be extended to compute the autocorrelation function (resp. the cross-correlation function) 
of second-order cyclostationary and cycloergodic processes (resp. second-order jointly cyclostationary and cycloergodic processes) as

$$
R_{x x}(t, \tau)=\langle x(t) x(t-\tau)\rangle_{T}=\lim _{K \rightarrow \infty} \frac{1}{2 K+1} \sum_{k=-K}^{K} x(t+k T) x(t+k T-\tau)
$$

(resp. $R_{y x}(t, \tau)$ with the first $x$ in the above substituted for $y$ ).

\subsection{Engine signals}

As far as engine signals are concerned, the assumption of stationarity has been used for a long time. Nevertheless, cyclostationarity seems more natural an assumption and it is surely better suited to reflect the properties of acoustical signals. This point is easily understood by considering the operation of an engine, which consists of the rotations and reciprocating motions of several mechanical components. Consequently, a series of non-stationary acoustical events are produced at specific instants within the engine cycle, and are then repeated from one cycle to the other. In the case of stationary operating conditions, the occurrence of such non-stationarities will be periodic and the corresponding acoustical signals will exhibit periodicity either in their waveform or, if somewhat random, at least in their energetic and spectral content-see Fig. 1. It turns out that the former type of behaviour is characteristic of first-order cyclostationarity and the latter to second-order cyclostationarity, both with main period $T_{\text {cycle }}$ equal to the engine cycle. A more detailed discussion on the factors leading to the cyclostationary property of engine signals may be found in Refs. $[3,8]$.

In concluding this section, it is important to outline that the cyclostationarity of engine signals is, strictly speaking, defined according to the angular variable $\theta$ that describes the rotation of the crankshaft and not according to the time variable $t$. However, as long as the operating conditions of the engine are assumed constant, there exists a perfect homothety between the angular and the time domains which can therefore be interchanged. How to cope with the slight operating fluctuations that may jeopardise that interchangeability will be discussed in the following section.

\section{The concept of cyclic sound intensity}

Of fundamental importance for characterising the noise radiated by engines is the measurement of sound intensity, i.e. the amount of acoustical energy that flows through a unit area per unit of time. When applied to cyclostationary signals, this leads to the appealing concept of cyclic sound intensity.

\subsection{Definitions}

As explained previously, engine signals are cyclostationary when measured during steady operating conditions. More particularly, a sound pressure signal $p(\mathbf{r}, t)$ and a particle velocity signal $v_{k}(\mathbf{r}, t)$ (where $k$ indicates the component in the

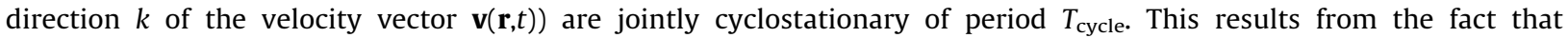
cyclostationarity is conserved through any linear operation (see [13]) such as Euler's equation. As a result, the corresponding component $I_{k}(\mathbf{r}, t)$ of the sound intensity vector $\mathbf{I}(\mathbf{r}, t)=p(\mathbf{r}, t) \mathbf{v}(\mathbf{r}, t)$ is also cyclostationary. In the same way, sound intensity inherits the cycloergodic property of signals $p(\mathbf{r}, t)$ and $v_{k}(\mathbf{r}, t)$. Thanks to these two properties, a time-synchronous average can then be performed with respect to the engine cycle $T_{\text {cycle }}$ so as to reach the expected value of $I_{k}(\mathbf{r}, t)$. This defines the $c y c l i c$ sound intensity as the averaged instantaneous sound intensity within an engine cycle:

$$
\mathbb{E}\left\{I_{k}(\mathbf{r}, t)\right\}=\left\langle p(\mathbf{r}, t) v_{k}(\mathbf{r}, t)\right\rangle_{T_{\text {cycle }}} .
$$

The above definition may now be easily generalised to other related acoustical quantities. To this end, let us introduce $\hat{p}(\mathbf{r}, t)=p(\mathbf{r}, t)+\mathrm{i} \mathcal{H}\{p(\mathbf{r}, t)\}$ and $\hat{v}_{k}(\mathbf{r}, t)=v_{k}(\mathbf{r}, t)+\mathrm{i} \mathcal{H}\left\{v_{k}(\mathbf{r}, t)\right\}$, the analytic signals computed from the Hilbert transform $\mathcal{H}\{\cdot\}$. Then,

$$
\begin{aligned}
I_{k}(\mathbf{r}, t)=p(\mathbf{r}, t) v_{k}(\mathbf{r}, t)= & \Re\{\hat{p}(\mathbf{r}, t)\} \Re\left\{\hat{v}_{k}(\mathbf{r}, t)\right\}=\frac{1}{4} \mathfrak{R}\left\{\left(\hat{p}(\mathbf{r}, t)+\hat{p}(\mathbf{r}, t)^{*}\right)\left(\hat{v}_{k}(\mathbf{r}, t)+\hat{v}_{k}(\mathbf{r}, t)^{*}\right)\right\} \\
= & \underbrace{\frac{1}{2} \mathfrak{R}\left\{\hat{p}(\mathbf{r}, t) \hat{v}_{k}(\mathbf{r}, t)^{*}\right\}}_{I_{k}^{\mathrm{a}}(\mathbf{r}, t)}+\underbrace{\frac{1}{2} \mathfrak{R}\left\{\hat{p}(\mathbf{r}, t) \hat{v}_{k}(\mathbf{r}, t)\right\}}_{I_{k}^{\mathrm{r}}(\mathbf{r}, t)},
\end{aligned}
$$

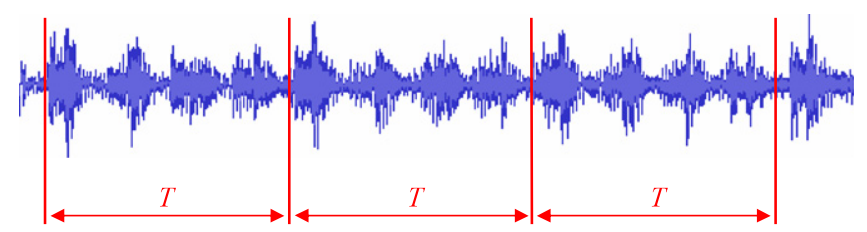

Fig. 1. Example of three cycles of a cyclostationary acoustical signal recorded in front of a 4-cylinder engine under steady operating conditions. Each cycle of period $T$ is composed of four bursts of energy corresponding to the successive combustions in the engine cylinders. 
where * denotes the complex conjugate. As discussed in Ref. [19], the first term in Eq. (10) reflects the instantaneous active intensity, i.e. that part of the acoustical energy flux that is effectively transferred along direction $k$. By complementarity, the second term must reflect the instantaneous reactive intensity, i.e. that part of the sound intensity with zero net transfer of energy along direction $k$. Now, applying the definition of the cyclic sound intensity to Eq. (10) yields

$$
\mathbb{E}\left\{I_{k}(\mathbf{r}, t)\right\}=\frac{1}{2}\left\langle\mathfrak{R}\left\{\hat{p}(\mathbf{r}, t) \hat{v}_{k}(\mathbf{r}, t)^{*}\right\}\right\rangle+\frac{1}{2}\left\langle\mathfrak{R}\left\{\hat{p}(\mathbf{r}, t) \hat{v}_{k}(\mathbf{r}, t)\right\}\right\rangle=\left\langle I_{k}^{\mathrm{a}}(\mathbf{r}, t)\right\rangle_{T_{\text {cycle }}}+\left\langle I_{k}^{\mathrm{r}}(\mathbf{r}, t)\right\rangle_{T_{\text {cycle }}} .
$$

This shows that, in general, the cyclic sound intensity decomposes as the summation of an active and a reactive part, which may be coined cyclic active intensity and cyclic reactive intensity, respectively. These are easily computed from their definitions in Eq. (11). The cyclic active intensity is representative of how the acoustical energy is periodically radiated from the engine, on a cyclic basis. In particular, the time-averaged intensity - which is so widely used in practice when the assumption of cyclostationarity is omitted - is then simply returned as the average value over one cycle,

$$
\overline{I_{k}^{a}}(\mathbf{r})=\lim _{T \rightarrow \infty} \frac{1}{T} \int_{-T / 2}^{+T / 2} I_{k}^{\mathrm{a}}(\mathbf{r}, t) \mathrm{d} t=\frac{1}{T_{\text {cycle }}} \int_{0}^{T_{\text {cycle }}}\left\langle I_{k}^{\mathrm{a}}(\mathbf{r}, t)\right\rangle_{T_{\text {cycle }}} \mathrm{d} t,
$$

thus clearly demonstrating that all time information is lost. On the other hand, the cyclic reactive intensity represents that part of the non-circulating energy, which may still repeat periodically on a cyclic basis. Note that, although the time-average value of the reactive intensity must be zero on a long-term basis, its instantaneous value on a cyclic basis is non-zero in general; yet its average value over one cycle necessarily vanishes since

$$
\overline{I_{k}^{r}}(\mathbf{r})=\lim _{T \rightarrow \infty} \frac{1}{T} \int_{-T / 2}^{+T / 2} I_{k}^{\mathrm{r}}(\mathbf{r}, t) \mathrm{d} t=\frac{1}{T_{\text {cycle }}} \int_{0}^{T_{\text {cycle }}}\left\langle I_{k}^{\mathrm{r}}(\mathbf{r}, t)\right\rangle_{T_{\text {cycle }}} \mathrm{d} t=0 .
$$

Finally, a last quantity of interest is the envelope of the cyclic reactive intensity which reflects the active intensity that the non-propagating wave would produce if its velocity were shifted by $\pi / 2$ so as to be in phase with pressure. Following Eq. (11), this is easily computed as

$$
\mathbb{E}\left\{I_{k}^{\text {env }}(\mathbf{r}, t)\right\}=\frac{1}{2}\left\langle\mathfrak{I}\left\{\hat{p}(\mathbf{r}, t) \hat{v}_{k}(\mathbf{r}, t)^{*}\right\}\right\rangle_{\text {cycle }},
$$

where the replacement of the real part by the imaginary part accounts for the $\pi / 2$ phase shift.

\subsection{Relevance of cyclic sound intensity}

The advantages of the cyclic sound intensity and its related quantities are multiple and mostly linked to the process of time-synchronous averaging.

\subsubsection{Cyclic sound intensity is an instantaneous quantity}

First, because the time-synchronous average returns a signal, the cyclic sound intensity enables the visualisation and the analysis of the instantaneous sound radiation as a function of time or crankshaft angle without losing the information contained in the very fast non-stationarities that would be otherwise erased or smoothed with classical time-averaging strategies based on the assumption of stationarity or quasi-stationarity. Therefore, the localisation of sound sources can be precisely achieved, with the best possible resolution, within the engine cycle. More precisely, assuming a nearly constant engine rotation $\Omega$ (in $\mathrm{Hz}$ ) and a temporal sampling frequency $F_{s}$, the ultimate angular resolution is

$$
\Delta \theta=360 \times \frac{\Omega}{F_{S}},
$$

where $\Delta \theta$ is in degrees. The resulting resolution is independent of the number $K$ of cycles that are averaged. To achieve a similar degree of averaging, other strategies will have to smooth the signal over $K$ consecutive angular samples which irreversibly results in a loss of angular resolution in the same proportion.

\subsubsection{Cyclic sound intensity is a periodic quantity}

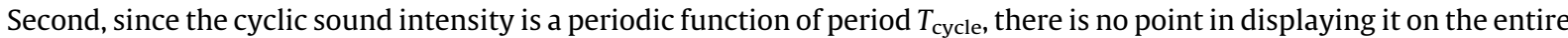
time record; only one representative cycle matters. This entails a substantial reduction of data in terms of display and storage.

\subsubsection{Cyclic sound intensity unambiguously decomposes into instantaneous active and reactive parts}

Third, as far as the physical interpretation of cyclic sound intensity in terms of its active and reactive parts is concerned, the process of time-synchronous averaging is crucial. Indeed, a reasonable amount of averaging is necessary for the reactive (resp. active) intensity to vanish in the case of purely propagating (resp. non-propagating) waves, thus making possible the separation between that part of the acoustical energy flux that is effectively conveyed by the engine to the far field and that part which is circulating in the near-field. Fig. 2 illustrates this point, where the cyclic active intensity measured $18 \mathrm{~cm}$ away from a diesel engine operating at $800 \mathrm{rev} / \mathrm{min}$, in the outward direction and in the third-octave band at $800 \mathrm{~Hz}$, is seen to be gradually building up in the positive direction as the number of cyclic averages increases, while the cyclic reactive part gradually vanishes. This clearly demonstrates that, on the average, the acoustical energy flux at that location and in that frequency range is essentially radiating outwards from the engine surface. Four intensity peaks are clearly identified, 
(a)

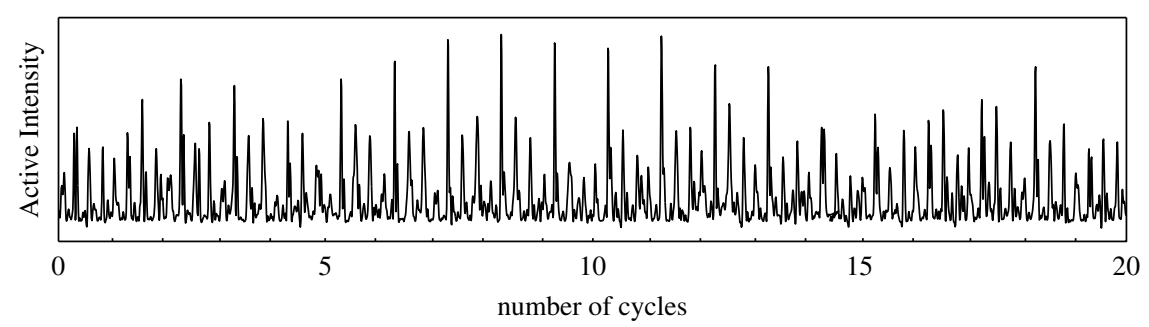

(b)

(c)
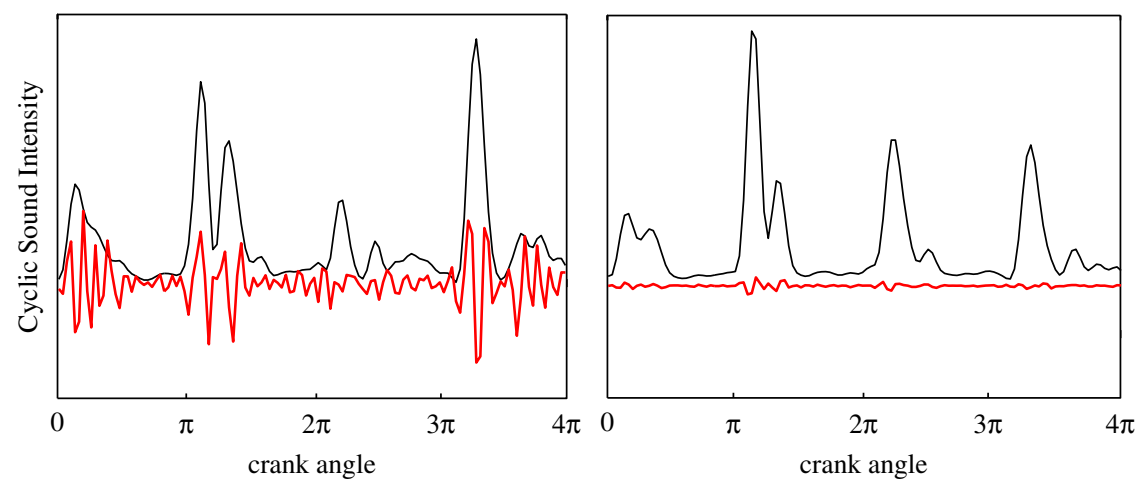

Fig. 2. Demonstration of the time-synchronous averaging effect in computing the cyclic sound intensity from probe signals recorded $18 \mathrm{~cm}$ from the centre of the top side of a 1998cc diesel engine. The engine speed is $800 \mathrm{rev} / \mathrm{min}$ and the intensity is displayed in the $800 \mathrm{~Hz} 1 / 3$-octave band. Thin line: active part; bold line: reactive part. (a) Sound intensity displayed over 20 engine cycles (no average), (b) time-synchronous average on three engine cycles, (c) timesynchronous average on 242 engine cycles.

separated by $180^{\circ}$, which corresponds to the sudden release of energy due to combustion in the four cylinders within one engine cycle.

As a final remark, it may be argued that the concept of active and reactive instantaneous intensities is somewhat ambiguous outside the stationary assumption because it involves a time-averaging operation - from a formal point of view, the reactive intensity is defined as the kernel of the time-averaging operator, and the active intensity as the complement of that kernel - which is in contradiction to the ability of extracting instantaneous features. However, by generalising the property of stationarity to a special case of non-stationarity, i.e. cyclostationarity, that ambiguity is circumvented by properly replacing the classical time-averaging operator by the time-synchronous averaging operator.

To sum up, cyclic sound intensity is found optimal on the one hand from a physical point of view by averaging energy over cycles and on the other hand from a calculation point of view by reducing the whole information over just one representative cycle. It offers the best time resolution among all possible "linear" averaging strategies-e.g. as compared to short-time or running averages. By comparison with other strategies that do not involve averaging at all, it enables a rigorous and unambiguous separation into active and reactive instantaneous intensity components.

\subsection{Practical aspects}

It is important to emphasise here that the cyclostationarity property of engine signals is, strictly speaking, fulfilled in the crankshaft-angle domain rather than in the time domain. This is because the periodicity of the non-stationarities - which defines cyclostationarity - is truly relative to the angular variable $\theta$ that describes the rotation of the crankshaft. It has been argued before that time and angle may be arbitrarily interchanged provided the operating conditions are constant. However, in practice slight fluctuations of the latter often occur that make the time period $T_{\text {cycle }}$ randomly vary around its mean value. Then, when described in the time domain, engine signals are no longer strictly cyclostationary but rather pseudocyclostationary. One simple solution to fix this trouble is to describe the signals in the angular domain, where they are truly cyclostationary of period $\Theta_{\text {cycle }}$, corresponding to two crankshaft revolutions, i.e. $720^{\circ}$. Thus, a resampling step from the time domain to the crankshaft angular domain is necessary before carrying out the time-synchronous average.

It has been argued that angular resampling may modify the spectral content of the signals in comparison with temporal sampling, because of frequency modulations induced by slight speed fluctuations. Although such an effect is true for sound pressure and particle velocity signals, it is virtually negligible on the cyclic sound intensity which is a non-oscillating signal-the time fluctuations of the energy flow are much slower than the waves which are carrying it. This may be formally quantified by recognising that speed fluctuations act as a low-pass filter (see [24]), the effect of which is all the more negligible as the signal is itself of a low-frequency nature. 


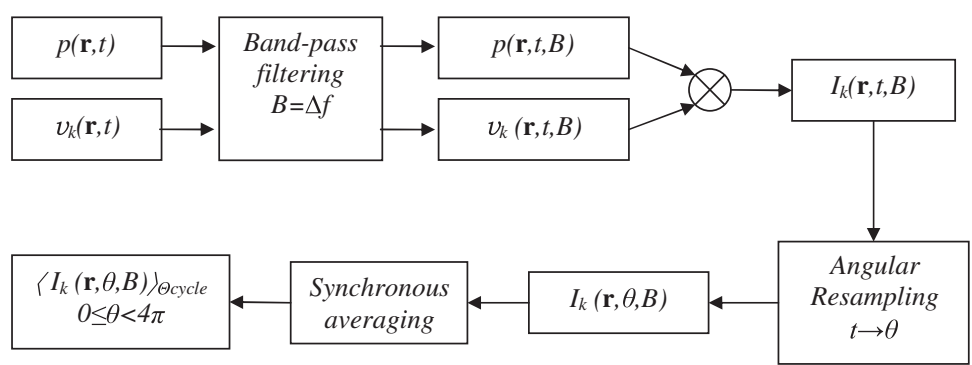

Fig. 3. Computation process of the cyclic sound intensity.

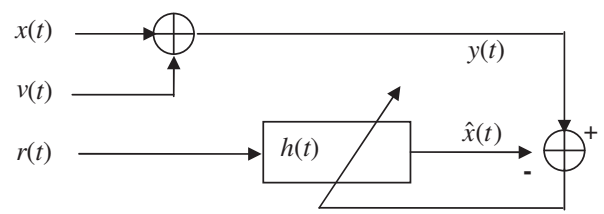

Fig. 4. Principle of Wiener filtering.

Finally, it is customary practice to display sound intensity in frequency bands, for instance such as returned by $1 / n$-octave band filters. This has a direct impact on the angular resolution of the cyclic sound intensity, that is the finest angular separation $\Delta \theta$ between two transients that can be assessed given a frequency bandwidth $B$. Namely, due to the uncertainty principle, the time and frequency resolutions are linked by the relationship

$$
\Delta t \cdot \Delta f \geq \frac{1}{4 \pi}
$$

which, in the angle domain, means

$$
\Delta \theta \cdot \Delta f \geq \frac{\Omega}{4 \pi}
$$

where $\Omega=\Delta \theta / \Delta t$ is the engine rotation speed.

The whole calculation process of the cyclic sound intensity, in the angular domain and in a frequency band $B$, is schemed in Fig. 3.

\section{Some applications of cyclic sound intensity in pre- and post-processing engine signals}

The cyclic sound intensity introduced hitherto is an attractive acoustical quantity as such. It is also particularly useful when used in conjunction with other specific space-time signal processing tools such as source separation and acoustical imaging, which are both enjoying novel developments in the case of cyclostationary engine signals.

\subsection{Cyclic sound intensity and source separation}

One of the breakthroughs permitted by the cyclostationarity property of engine signals is the improvement of noise source separation techniques, in particular by using the so-called "cyclic Wiener filter" whose principle is shortly reminded hereafter [16-18].

The aim of the Wiener filter is to separate a noisy measurement

$$
y(t)=x(t)+v(t)
$$

into its contribution $x(t)$ from a specific source and the remaining "noise" $v(t)$. This has been traditionally achieved by using a reference signal $r(t)$, strongly coherent with the source of interest while uncorrelated with all other interfering sources and masking noise embodied in $v(t)$, such that $x(t)$ may be estimated from the filtering operation:

$$
\hat{x}(t)=\sum_{\tau} h(\tau) r(t-\tau)
$$

as illustrated in Fig. 4. The best linear (Wiener) filter $h(t)$ intervening in Eq. (19) is then found by minimising the quadratic error:

$$
h(\tau)=\underset{h}{\operatorname{argmin}} \mathbb{E}\left\{|y(t)-\hat{x}(t)|^{2}\right\}, \quad \forall t .
$$


In this classical formulation, having the Wiener filter linear and time-invariant is optimal under the stationary assumption of signals $x(t)$ and $v(t)$. However, this is no longer true when the separation is to be applied to cyclostationary signals. In this specific case, the optimal Wiener filter is shown to be linear and periodic,

$$
\hat{x}(t)=\sum_{\tau} h(t, \tau) r(t-\tau)
$$

where $h(t, \tau)=h\left(t+T_{\text {cycle }}, \tau\right)$ is the filter impulse response at time $t$ to an impulse at time $t-\tau$. Here, the time dependence of the optimal filter is fundamental to be able to extract the fast non-stationarities - such as combustion noise or mechanical impacts - with rapidly changing spectral contents and energy magnitudes within the engine cycle. This is best understood when time and angle can be interchanged under the assumption of steady operating condition, in which case

$$
\hat{x}(t)=\sum_{\tau} h(\theta(t), \tau) r(t-\tau)
$$

where the filter $h(\theta, \tau)=h\left(\theta+\Theta_{\text {cycle }}, \tau\right)$ adjusts itself to each angle $\theta$ in the engine cycle. The estimation of the cyclic Wiener filter $h(t, \tau)$ is found by minimising the same quadratic error as in Eq. (20), i.e.

$$
h(t, \tau)=\underset{h}{\operatorname{argmin}} \mathbb{E}\left\{|y(t)-\hat{x}(t)-\mathbb{E}\{y(t)-\hat{x}(t)\}|^{2}\right\}, \quad \forall t,
$$

where the expected value operator $\mathbb{E}$ is now to be understood in the sense of Eq. (7) and where care has been taken to remove the intercept $\mathbb{E}\{y(t)-\hat{x}(t)\}$ signal - this is no longer a constant value of time under the cyclostationary assumption - that would otherwise interfere with the estimation process [18]. Substituting $\hat{x}(t)$ for its expression in Eq. (22) and expanding, the solution to Eq. (23) is then returned by the linear system of equations,

$$
C_{y r}(t, \tau)=\sum_{\tau^{\prime}} h\left(t, \tau^{\prime}\right) C_{r r}\left(t-\tau^{\prime}, \tau-\tau^{\prime}\right), \quad \forall \tau,
$$

where

$$
C_{y r}(t, \tau) \hat{=} R_{y r}(t, \tau)-m_{y}(t) m_{r}(t-\tau)
$$

is the cross-covariance between signals $y(t)$ and $r(t)$-and similarly for $C_{r r}(t, \tau)$ with $y$ replaced by $r$. Upon invoking the assumption of cycloergodicity, these quantities are easily computed from time-synchronous averages on the measured signals $y(t)$ and $r(t)$, as explained in Section 2 .

It is emphasised at this junction that the cyclic Wiener filter is likely to achieve a better separation than other classical "referenced techniques", not only because it is apt to extract very fast transient signals as being a function of $\theta$, but also because it is unaffected by periodic interferences. By construction - and rather uniquely to the author's knowledge - it removes the effect of first-order cyclostationarity corresponding to common harmonics of rotation that are shared by the reference and the noise signals, yet without an implicit causality link between them. By exploiting pure second-order cyclostationarity only - as embodied by the cross-covariance definition of Eq. (25) - it then guaranties that correlation between the measured signal $y(t)$ and the reference signal $r(t)$ implies physical causation.

Eventually, when applied jointly to sound pressure and particle velocity signals, the cyclic Wiener filter comes out as a useful pre-processing tool for separating the cyclic sound intensity into that part due to a source contribution and that part due to remaining additive noise. Namely,

$$
\left\langle I_{y, k}(\mathbf{r}, t)\right\rangle_{T_{\text {cycle }}}=\left\langle I_{x, k}(\mathbf{r}, t)\right\rangle_{T_{\text {cycle }}}+\left\langle I_{v, k}(\mathbf{r}, t)\right\rangle_{T_{\text {cycle }}},
$$

where $\left\langle I_{y, k}(\mathbf{r}, t)\right\rangle_{T_{\text {cycle }}}$ is defined as in Eq. (9) with $\mathbf{y}(t)=\left\{p(t), v_{k}(t)\right\}^{T}$, and similarly for $\left\langle I_{x, k}(\mathbf{r}, t)\right\rangle_{T_{\text {cycle }}}$ and $\left\langle I_{v, k}(\mathbf{r}, t)\right\rangle_{T_{\text {cycle }}}$ with

$$
\hat{\mathbf{x}}(t)=\left\{\begin{array}{c}
p_{x}(t) \\
v_{x, k}(t)
\end{array}\right\}=\sum_{\tau}\left\{\begin{array}{l}
h_{r p}(t, \tau) \\
h_{r v}(t, \tau)
\end{array}\right\} r(t-\tau),
$$

and $\boldsymbol{v}(t)=\mathbf{y}(t)-\hat{\mathbf{x}}(t)$, respectively. Note that only one reference signal $r(t)$ for the source of interest is necessary - yet with two different cyclic Wiener filters $h_{r p}$ and $h_{r v}$ - to achieve the separation on both the sound pressure and the particle velocity signals.

\subsection{Cyclic sound intensity movies and acoustical imaging}

The analysis of the sound intensity field, as obtained from post-processing the data collected from scanning probes or microphone arrays, is extremely useful in acoustical imaging techniques to localise and identify the sources and sinks of energy over the surface of an object. The cyclic sound intensity field may just as well be visualised during an engine cycle by means of a sound radiation movie, whose each frame $\left\langle I_{k}(\mathbf{r}, \theta, B)\right\rangle_{\Theta_{\text {cycle }}}$ corresponds to a sound radiation map calculated at a specific crank angle $\theta$ and in a specific frequency band $B$. This will not only enable the localisation of the radiation areas in space, but it will also allow the determination of their occurrence precisely in angle as well as the appraisal of their evolution within the engine cycle. Compared to traditional "stationary" approaches, this will provide the user with considerably more information, thus leading to better localisation, identification, and assessment of the structure of the noise sources.

All these points will now be illustrated on some experimental examples. 


\section{Experimental results}

The following industrial examples illustrate the use of the cyclic sound intensity for the applications discussed in the previous section. The data come from sound intensity probe measurements and near-field-acoustical-holography (NAH) measurements carried out on two different Renault dCi 4-stroke diesel engines in a semi-anechoic room. In both cases, signals were recorded during about 150 engine cycles and sampled at $16384 \mathrm{~Hz}$. All sound radiation maps are displayed hereafter with a $10 \mathrm{~dB}$ dynamic range.

\subsection{Source separation results}

In this first experiment, the objective was to compute the cyclic sound intensity over the surface of the first engine and to separate it into its contribution from the combustion sources and its contribution from the remaining "mechanical" sources. Therefore, a set of cyclic Wiener filters was applied to the sound pressure signals returned by the scanned intensity probe placed at $10 \mathrm{~cm}$ from the distribution side of the engine with a $12 \mathrm{~mm}$ spacing between the microphones. The reference signals used in the cyclic Wiener filters were the cylinder pressures measured inside the four cylinders. The separated signals at each measurement point were then used to compute the cyclic sound intensity and, proceeding in a similar way for each of the 80 measurement points, cyclic sound intensity maps could be obtained. The overall active cyclic sound intensity field averaged over one period as in Eq. (12) - is displayed in Fig. 5(a) in the $1250 \mathrm{~Hz} 1 / 3$-octave band for an engine speed of $800 \mathrm{rev} / \mathrm{min}$. Fig. 5(b) and (c) displays the cyclic sound intensity fields due to the contribution of the combustion sources and the mechanical sources, only and, respectively. These results are very interesting in understanding the origins and the transfers of noise. It is seen from Fig. 5(b) that the sound radiated from the catalytic pre-converter (on the top left corner of the engine face) and from the crankshaft pulley (on the bottom centre of the face) originates from combustion sources, which is explained by the structure-borne propagation of noise from the cylinders to the cranktrain. Fig. 5(c) shows that the other

(a)

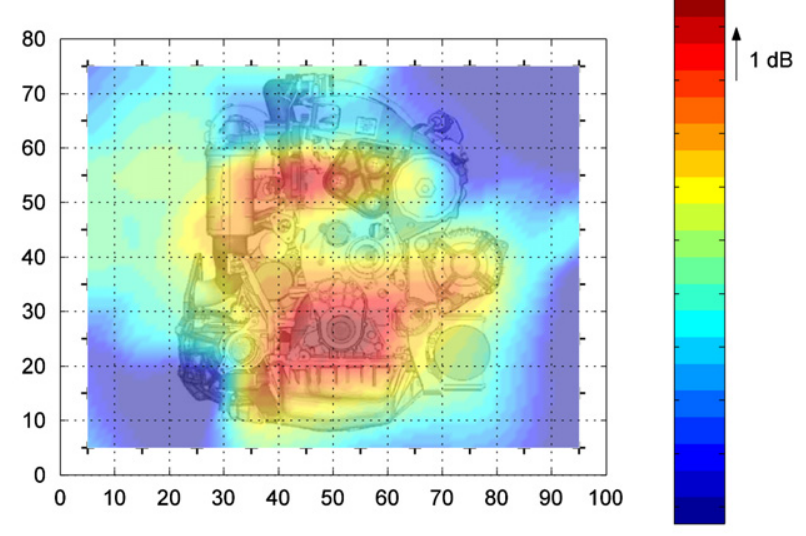

(b)

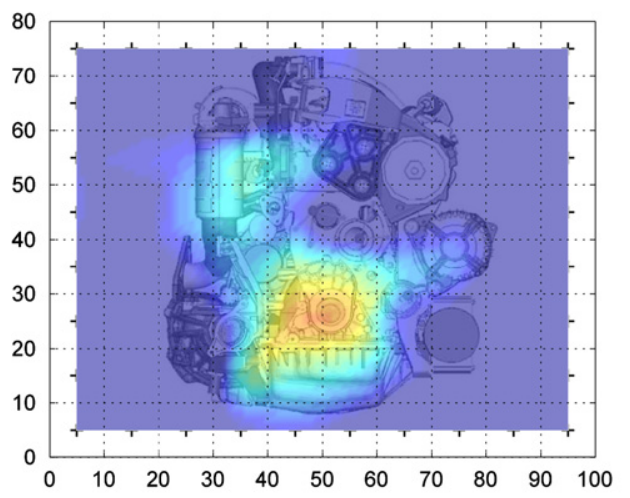

(c)

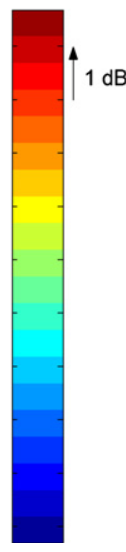

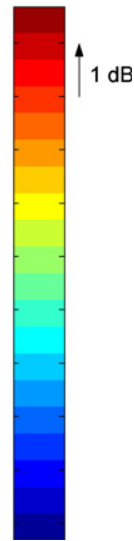

Fig. 5. Separation of the overall active cyclic sound intensity, averaged over one engine cycle, radiated by the distribution side of a 1870 cc diesel engine into a contribution from the combustion source and a contribution from the mechanical noise. The engine speed is $800 \mathrm{rev} / \mathrm{min}$ and the active sound intensity is displayed in the $1250 \mathrm{~Hz}$ 1/3-octave band. (a) Overall active cyclic sound intensity, (b) contribution of the combustion source, (c) contribution of the mechanical noise. (The reader's attention is attracted to the fact that scales are in $\mathrm{dB}$, the reason why map (b) superimposed on map (c) may not result exactly in map (a), although additivity exactly holds on a linear scale.) 
sound radiation areas are due to mechanical sources localised near the alternator (on the bottom right corner of the engine face) and on the distribution rollers (at the top of the engine face).

Fig. 6 compares the results of noise source separation returned by different methods. The bar chart represents the overall sound power level (in $\mathrm{dB}$ ) radiated from the exhaust side of the engine operating at $3000 \mathrm{rev} / \mathrm{min}$ at full charge. The global sound power is calculated by multiplying the active cyclic sound intensity averaged over one engine cycle, as obtained in Fig. 5(a), with surface elements corresponding to the measurement points and then summing over the whole surface. This quantity is completely equivalent to the classical sound power calculated by the classical sound intensity according to Eq. (12). The solid line refers to the combustion level estimated by a commercial solution using a classical sound intensitybased calculation, whereas the dotted line is that returned by cyclic Wiener filtering. Fig. 6 shows that the commercial solution clearly overestimates the combustion contribution which is unrealistically higher than the overall level. This is because the commercial solution estimates the combustion level from the "stationary" coherence between the raw signals measured by each microphone of the acoustical array and the cylinder pressure signals measured inside the four cylinders,

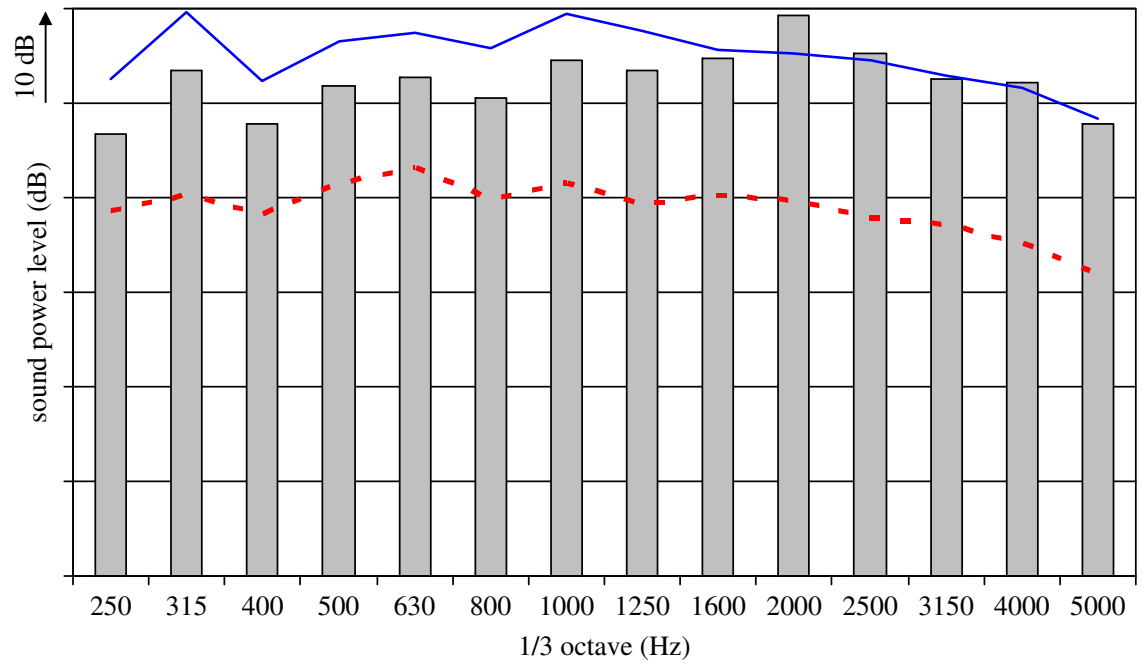

Fig. 6. Sound power emitted by an exhaust face engine operating at $3000 \mathrm{rev} / \mathrm{min}$ at full charge. Bar chart: overall level; solid line: power contribution from combustion sources returned by a commercial solution; dotted line: power contribution from combustion source returned by the cyclic Wiener filter.

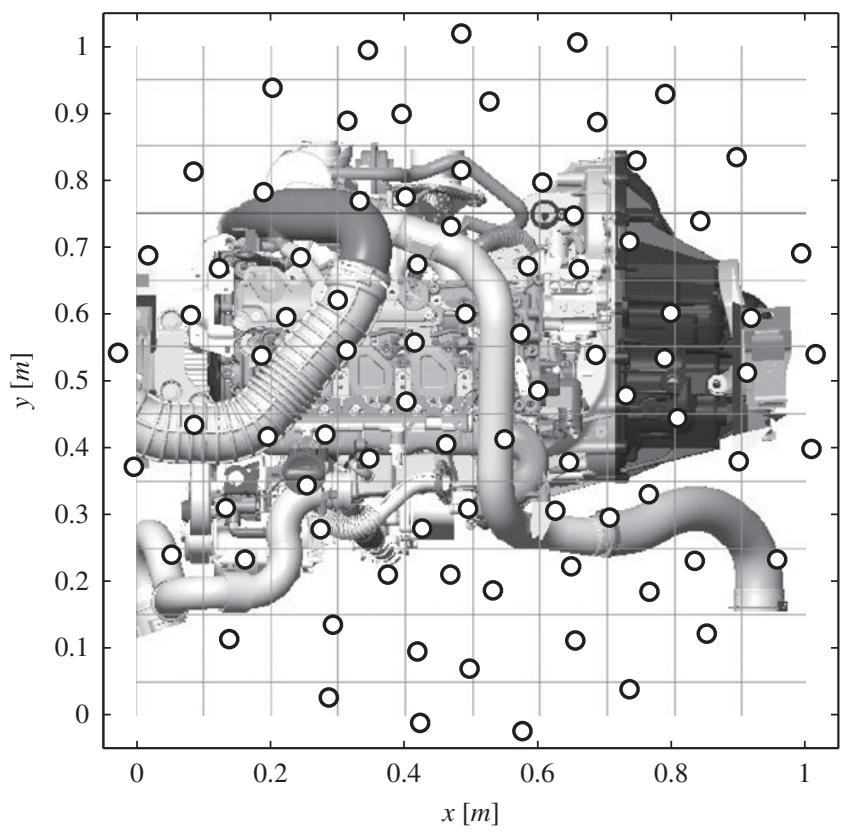

Fig. 7. Scheme of the 84-microphone irregular array with uniform density used for the MAH measurements. Each spot corresponds to a microphone position. 
as initially developed by Bendat and Piersol [20]. The global combustion contribution is then obtained by summing the combustion contributions of each cylinder. Unfortunately, the combustion noise of the cylinders are coherent between each other in the low-frequency domain. As a consequence, what is considered as coherent with cylinder 1 is also coherent with cylinders 2-4 and the summation of the four quantities overestimates the actual level. On the other hand, the combustions in the cylinders are not coherent between each other any more in the high-frequency domain. However, the estimated level of the combustion contribution is also unrealistic. The more realistic reason is that in this case, the contributions of the four cylinders contain additional noise sources that are coherent with the combustion noise and then erroneously added to the real combustion contributions. The cyclic Wiener filter does not suffer from any of those shortcoming because it is based on the pure second-order cyclostationarity property only - i.e. on signals after removal of periodic components - whereas the commercial solution cannot make this distinction $[12,18]$.

Similar results were obtained from NAH and beamforming measurements by applying, in the same way, a separation step on the sound pressure signals recorded by an antenna of microphones. Fig. 7 displays a scheme of the 84-microphone irregular array with uniform density used for the NAH measurements. The measurements were conducted synchronously since this array could cover a surface equivalent to the engine (however, the scanning technique could also be applied provided that additional microphones, placed at fixed locations, are used to keep the phase information that is as well preserved after the application of the cyclic Wiener filter (see Appendix A)). The array was placed $18 \mathrm{~cm}$ in front of the top side of the second engine operating at a speed of $800 \mathrm{rev} / \mathrm{min}$. Fig. 8 displays, in the $1250 \mathrm{~Hz} 1 / 3$-octave band, the source

(a)

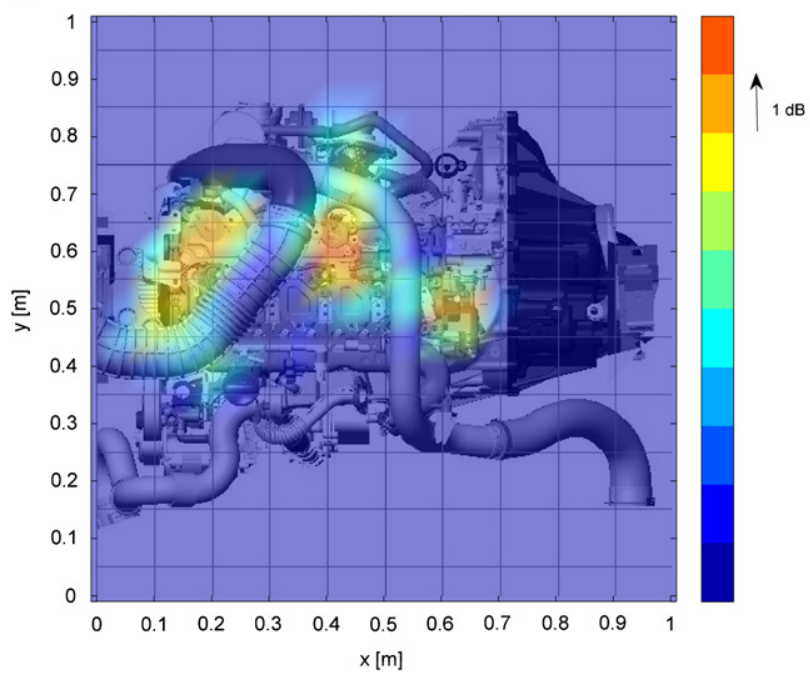

(b)

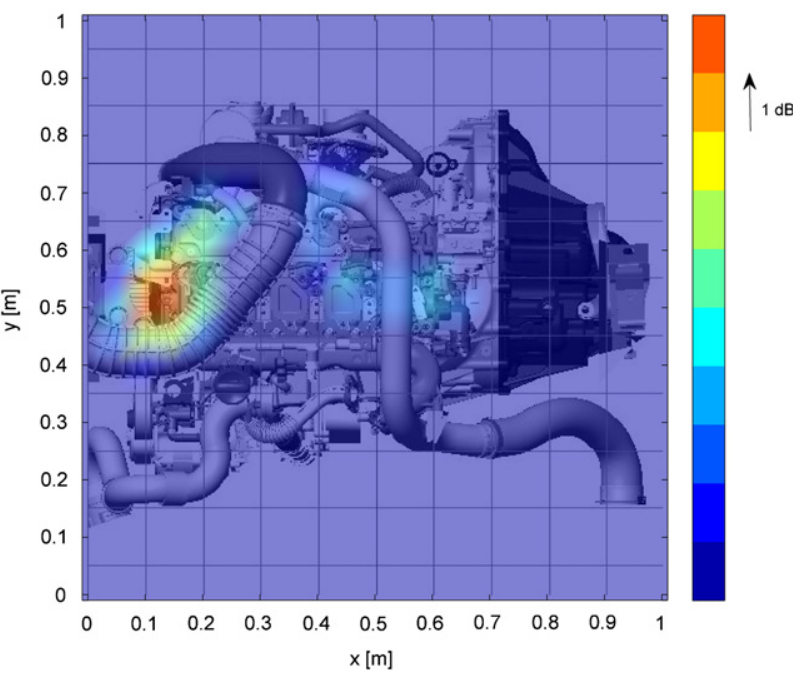

(c)

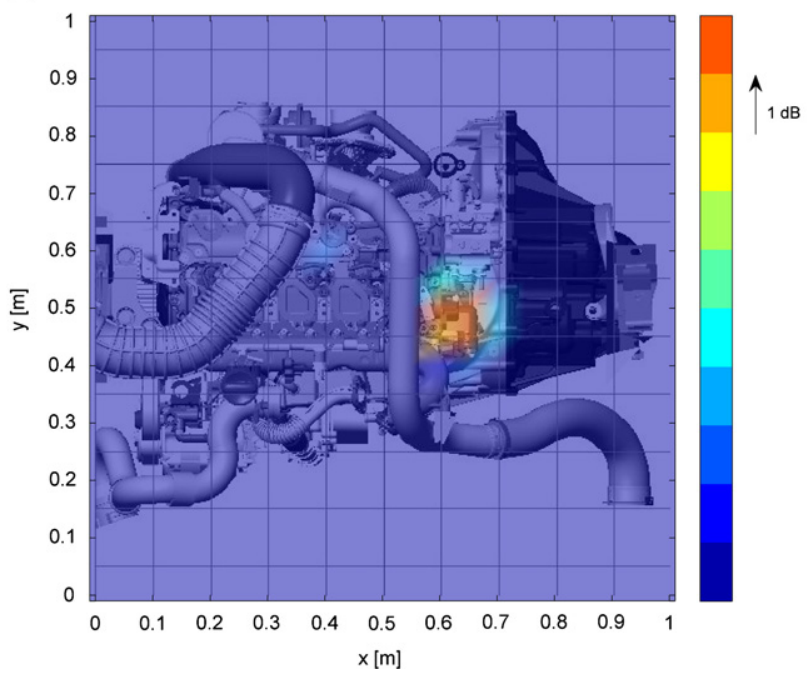

Fig. 8. Separation of the overall sound intensity radiated by the top side of a 1998cc diesel engine into a contribution from the combustion source and a contribution from the injection noise. The engine speed is $800 \mathrm{rev} / \mathrm{min}$ and the active sound intensity is displayed in the 1250 Hz 1/3-octave band. (a) Overall sound intensity, (b) contribution of the combustion source, (c) contribution of the injection noise. 

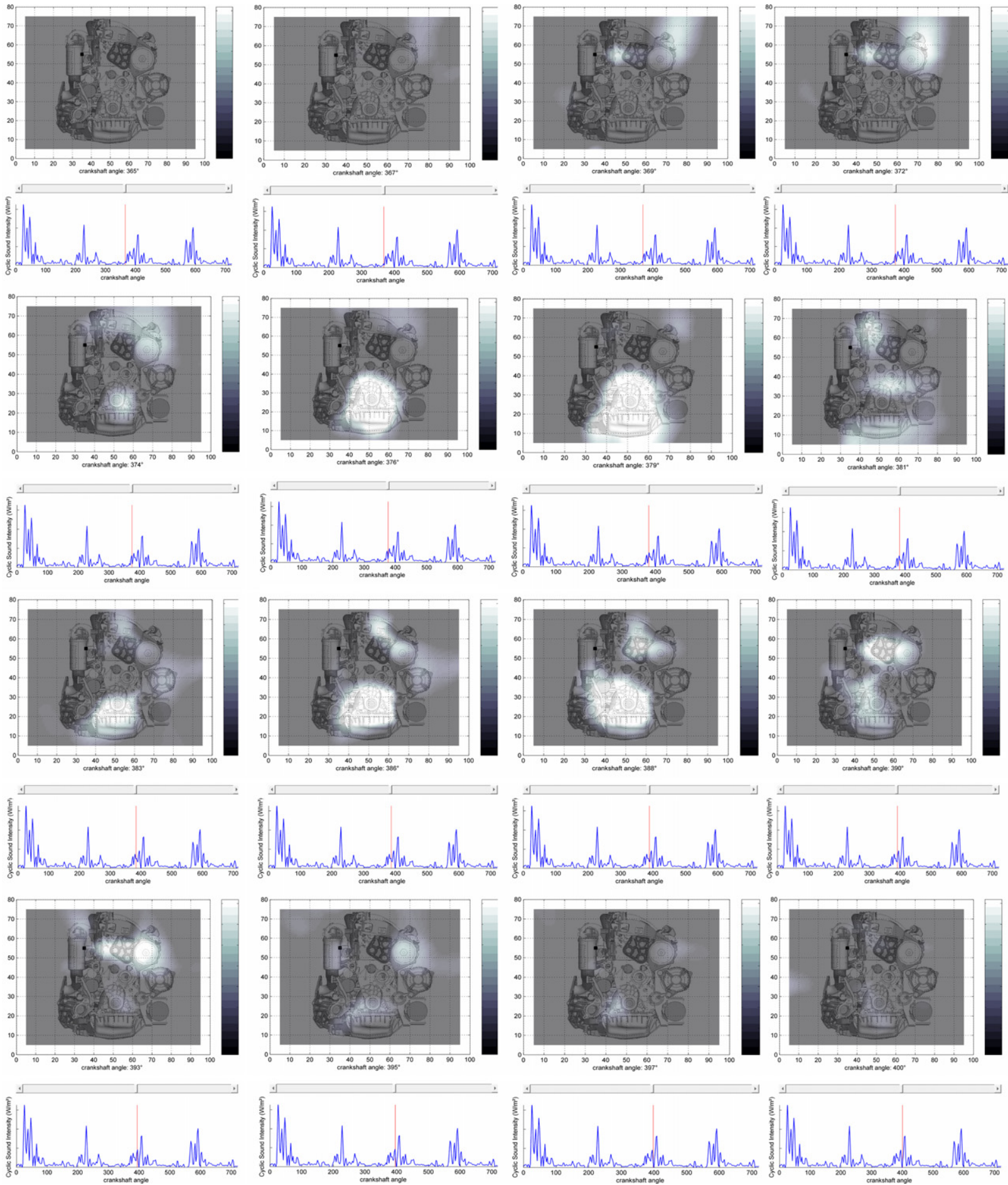

Fig. 9. Consecutive frames composing a cyclic sound intensity movie, showing how the acoustical energy flux is evolving as a function of the crank angle $\left(\Delta \theta=2.5^{\circ}\right)$ on the distribution side of a diesel engine operating at $800 \mathrm{rev} / \mathrm{min}$, in the $1000 \mathrm{~Hz} 1 / 3-\mathrm{octave}$ band.

separation results performed on these latter measurements. In this case, two sets of reference signals were available to separate the contribution of (i) the combustion noise, (ii) the injection noise, and (iii) the residual mechanical noise. The first set of reference signals was provided by cylinder pressure measurements as done in the previous experiment, and the second set by vibration measurements on the pipes linking the common rail to the injectors. The separation step was then performed in cascade to first separate the combustion part from the pressure signals and then the injection part from the residual signals. 

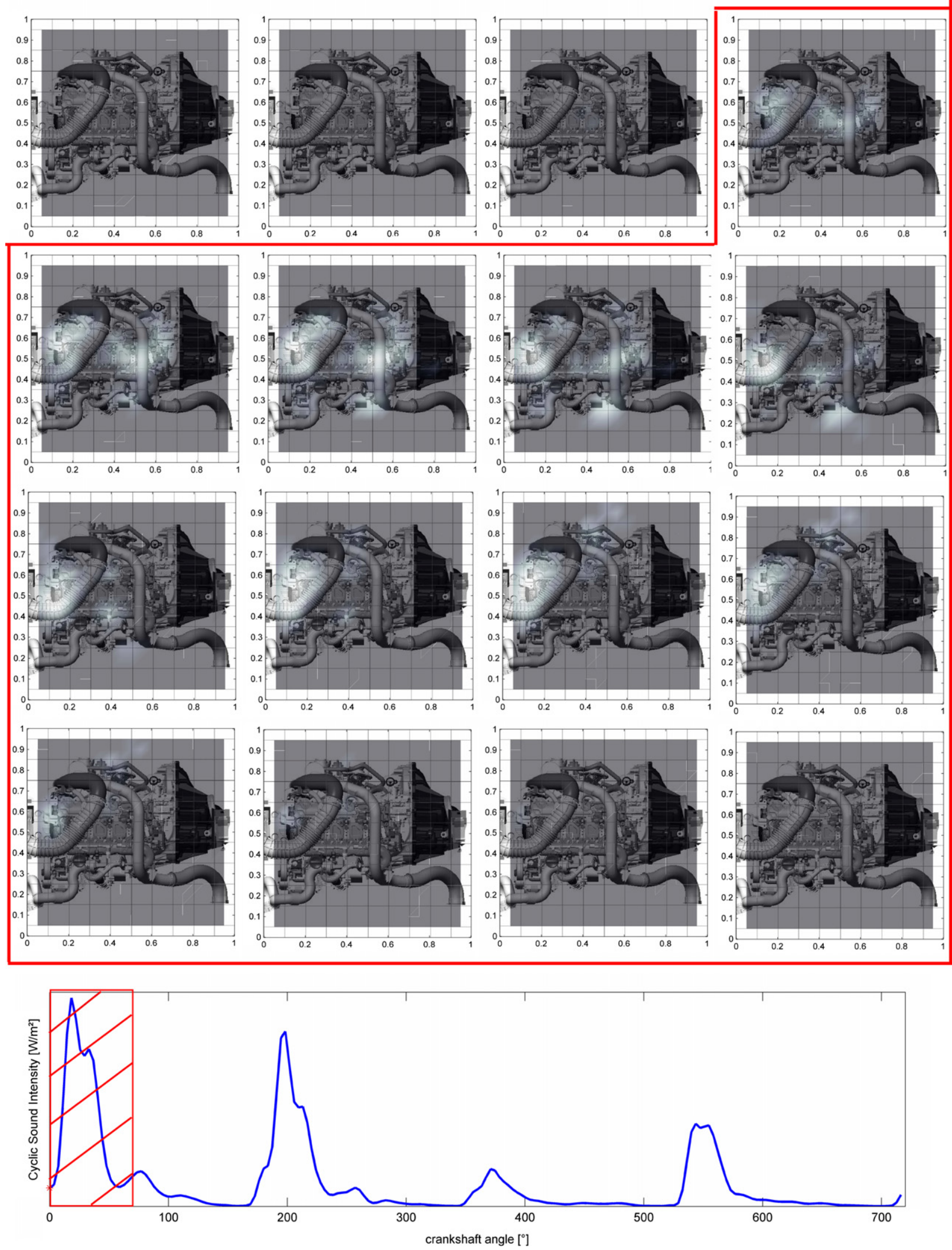

Fig. 10. Consecutive frames composing a cyclic sound intensity movie, showing how the combustion part of the acoustical energy flux is evolving as a function of the crankshaft angle $\left(\Delta \theta=3.7^{\circ}\right)$ on the top side of a diesel engine operating at $800 \mathrm{rev} / \mathrm{min}$, in the $1250 \mathrm{~Hz} 1 / 3-\mathrm{octave}$ band. The frames correspond to the hatched area of the cyclic sound intensity on the engine top side displayed in the bottom panel. 
After applying this process to each microphone, an NAH retropropagation step was undertaken to obtain sound radiation maps in a tangent plane to the engine face. The results are displayed in Fig. 8(a)-(c). Here again, quite interesting results are arrived at. After separation, the hot areas of sound radiation identified in Fig. 8(a) are unambiguously assigned to the combustion sources on the left side of the engine and to the injection source on the right side. This completely agrees with the physics since the left area is above the distribution side and the right area is just above the injection pump.

\subsection{Cyclic sound intensity results}

As previously explained, the cyclic sound intensity field may be conveniently displayed as a movie during the engine cycle with the definite advantage of bringing additional information on the localisation of sources in angle, space and frequency. Fig. 9 is an extract of the frames forming such a movie from crankshaft angles $365-400^{\circ}$ with a step of $2.5^{\circ}$, around the sector where the third combustion within the engine cycle takes place. Each frame comprises two parts. The lower part is the cyclic sound intensity curve calculated for a specific measurement point and the upper part is the instantaneous sound radiation map at the crankshaft angle corresponding to the position of the cursor (the vertical line) on the lower graph.

Similarly, a movie of the contribution of a specific source on the sound intensity can be obtained by coupling noise source separation with cyclic sound intensity calculation. Fig. 10 shows such an example where the contribution of the combustion source on the cyclic sound intensity field is displayed as a movie. The movie extract consists of 16 consecutive frames, from crankshaft angles $0-64^{\circ}$ with a step of $4^{\circ}$ corresponding to the hatched area of the cyclic sound intensity signal calculated over the entire top side of the engine (see bottom panel of Fig. 10). The evolution of the sound radiation due to the first combustion can be perceived from the 4th frame to the last one. The high sound radiation areas fully correspond to the former sound radiation area noticed on the combustion part of the sound intensity displayed in Fig. 8(b). The computation of such a movie after the NAH treatment is possible since cyclostationarity is preserved (see Appendix B). Technically speaking, this computation was obtained from NAH measurements as previously described in Section 5.1, as an additional step after retropropagation from the measurement surface to the hologram surface. An inverse Fourier transform was then performed in order to come back to the time domain, where care was taken to avoid wrap-around errors due to the circular convolution by using the overlap-and-add technique [21-23].

\section{Conclusions}

The present study intended to demonstrate the advantages of a newly defined acoustical quantity: the cyclic sound intensity. Associated to acoustical imaging techniques, the cyclic sound intensity makes possible the calculation of instantaneous sound radiation movies that precisely point to the angle of occurrence, inside an engine cycle, of particular noise sources. Radiation of noise sources can then be localised in space, frequency and time (crankshaft angle). Furthermore, when coupled with cyclic Wiener filtering, it enables the separation of the global radiated sound field into its various contributions, thus offering a better understanding of how noise propagates through the structure.

\section{Acknowledgment}

The authors would like to thank the company RENAULT for funding this research work conducted in its Powertrain NVH department.

\section{Appendix A. Cycling Wiener filtering and phase synchronization}

The NAH separation results presented in Section 5 of the paper have been obtained from signals synchronously recorded by the microphones of an acoustic array. Similar results are conceptually possible with scan-based NAH, as demonstrated in this section. In that case, it is important to specify that the phase synchronization is preserved after the source separation step by cyclic Wiener filtering in the coupling process.

Let $\varpi_{i}$ refer to the $i$-th scanning experiment, leading to the measured pressure signal $p\left(\mathbf{r}_{i}, t ; \varpi_{i}\right)$ at position $\mathbf{r}_{i}$. Let also $r_{k}\left(t ; \varpi_{i}\right)$, be $k=1, \ldots, R$ reference signals measured simultaneously and systematically for each scanning experiment by a set of $R$ microphones. $M$ scanning experiments then lead to the $M$ sets of signals

$$
\left\{p\left(\mathbf{r}_{i}, t ; \varpi_{i}\right) ; r_{k}\left(t ; \varpi_{i}\right)\right\}, \quad i=1, \ldots, M, k=1, \ldots, R .
$$

Suppose now that one single experiment is conducted (whose label $\varpi$ is therefore omitted), where all pressure signals $p\left(\mathbf{r}_{i}, t\right)$ are measured simultaneously by a microphone array together with reference signals on the cylinder pressures which reflect the combustion sources, as described in the paper. Let us take the case of a single reference signal, $r(t)$, for simplicity. Then, as explained in the paper, the prediction of $p\left(\mathbf{r}_{i}, t\right)$ from $r(t)$ returns an estimate of the combustion noise, say

$$
p_{x}\left(\mathbf{r}_{i}, t\right)=\int h_{r p_{i}}(t, \tau) r(t-\tau) \mathrm{d} \tau,
$$


which involves the periodically time-varying Wiener filter $h_{r p_{i}}(t, \tau)=h_{r p_{i}}(t+T, \tau)$. In particular, the predicted combustion noise is seen to yield the cross-correlation

$$
\mathbb{E}\left\{p_{x}\left(\mathbf{r}_{i}, t\right) p_{x}\left(\mathbf{r}_{j}, t^{\prime}\right)\right\}=\iint h_{r p_{i}}(t, \tau) h_{r p_{j}}\left(t^{\prime}, \tau\right) \mathbb{E}\left\{r(\tau) r\left(\tau^{\prime}\right)\right\} \mathrm{d} \tau \mathrm{d} \tau^{\prime},
$$

where phase synchronisation between $p_{x}\left(\mathbf{r}_{i}, t\right)$ and $p_{x}\left(\mathbf{r}_{j}, t\right)$ is perfectly retained for any $(i, j)$ since it is expressed as a function of the (non-identically zero) autocorrelation $\mathbb{E}\left\{r(\tau) r\left(\tau^{\prime}\right)\right\}$. Now, it must remember that in the case of cyclostationary signal, the cross-correlations

$$
\begin{gathered}
R_{p_{x i} p_{x j}}(t, \tau)=\left\{p_{x}\left(\mathbf{r}_{i}, t\right) p_{x}\left(\mathbf{r}_{j}, t^{\prime}\right)\right\}, \quad \tau=t-t^{\prime} \\
=R_{p_{x i} p_{x j}}(t+T, \tau)
\end{gathered}
$$

are periodic functions of time $t$ and, as such, can easily be synchronised w.r.t the engine cycle by use of an encoder, as explained above. Of course, this requires the proper implementation of the mathematical operator $\mathbb{E}$ on the cycles, according to Eq. (9), so that time information $t$ is not lost-as it would be otherwise if classical time-averaging was used. Under this condition, the quadratic errors in Eq. (23) - whose definition is based on $\mathbb{E}$ - is guaranteed to keep time information (synchronisation).

\section{Appendix B. Cyclostationarity and NAH}

This subsection proves that the cyclostationarity property of raw signals is preserved when passing through the SONAH filter representing the back propagation onto the hologram surface.

Let $x(t)$ be a second-order cyclostationary signal with cyclic period $T$, i.e. such that its correlation is periodic,

$$
R_{x x}(t, \tau)=\mathbb{E}\left\{x(t) x(t-\tau)^{*}\right\}=R_{x x}(t+T, \tau),
$$

which is input into the sequence of transformations. Now let us decompose $x(t)$ in a series of snapshots

$$
x(t)=\sum_{k} x_{w_{k}}(t)=\sum_{k} x(t) w(t-k \Delta), \quad \sum_{k} w(t-k \Delta)=1,
$$

where $w(t)$ is a smooth data window of finite duration over an interval $\left[0, T_{w}\right]$ that is shifted by equidistant increments along the signal so as to select snapshots $x_{w_{k}}(t)$. Let $X_{w_{k}}(\omega)$ be the (short-time) Fourier transform of snapshot $x_{w_{k}}(t)$, i.e.

$$
X_{w_{k}}(\omega)=F\left\{x_{w_{k}}(t)\right\}=\int_{k \Delta}^{k \Delta+T_{w}} x_{w_{k}}(t) \exp ^{-j \omega t} \mathrm{~d} t
$$

where integration is carried out over the support set of $w(t-k \Delta)$. Let $H(\omega)$ be the frequency response of an inverse operator that symbolises the SONAH retropropagation (spatial information is not accounted for here since in it has no effect on the validity of the proof). Hence, the sequence of transformations up to SONAH processing produces the Fourier transform

$$
Y_{w_{k}}(\omega)=X_{w_{k}}(\omega) H(\omega) \text {. }
$$

Finally the output signal in the time domain is obtained by inverse Fourier transforming Eq. (B.4),

$$
y_{w_{k}}(t)=\frac{1}{2 \omega} \int Y_{w_{k}}(\omega) \exp ^{j \omega t} \mathrm{~d} \omega=\int h(\tau) x_{w_{k}}(t-\tau) \mathrm{d} \tau,
$$

which is the convolution of snapshot $x_{w_{k}}(t)$ with the impulse response $h(t)=\Phi^{-1}\{H(\omega)\}$. The full-length signal is then reconstructed by "inversing” Eq. (B.2),

$$
y(t)=\sum_{k} y_{w_{k}}(t)
$$

Now, the correlation of $y(t)$ is

$$
R_{y y}(t, \tau)=\mathbb{E}\{y(t) y(t-\tau)\}=\iint h(\tau) h\left(\tau^{\prime}\right) \mathbb{E}\left\{x(t) x\left(t-\tau^{\prime}\right)\right\} \mathrm{d} \tau \mathrm{d} \tau^{\prime}=\iint h(\tau) h\left(\tau^{\prime}\right) \mathbb{E}\left\{x(t+T) x\left(t+T-\tau^{\prime}\right)\right\} \mathrm{d} \tau \mathrm{d} \tau^{\prime}=R_{y y}(t+T, \tau),
$$

where Eq. (B.5), resp. (Eq. (B.4)) have been used to establish the second resp.(third) equality. Since $R_{y y}(t, \tau)=R_{y y}(t+T, \tau)$ is periodic, $y(t)$ is second-order cyclostationary with cyclic period $T$ just as $x(t)$ is. As a consequence, the cyclostationarity property is preserved after back propagation.

Note: the decomposition of the signals into snapshot as detailed in Eq. (B.2) is standard in array processing. It is necessary for at least two reasons. First, it involves the computation of many short-time Fourier transforms instead of a very long one, which is computationally more efficient when Eq. (B.3) is implemented with the FFT. Second, it limits the computation of the inverse frequency response function $H(\omega)$ of SONAH (which is a costly process) to only a limited number of frequency lines equidistant by $1 / \Delta$; therefore, the shorter the snapshot, the lower the computational cost of SONAH. 


\section{References}

[1] E. Serpedin, F. Panduru, I. Sari, G.B. Giannakis, Bibliography on cyclostationarity, Signal Processing 85 (12) (2005) $2233-2303$.

[2] W.A. Gardner, A. Napolitano, L. Paura, Cyclostationarity: half a century of research, Signal Processing 86 (4) (2006) $639-697$.

[3] J. Antoni, J. Danière, F. Guillet, Effective vibration analysis of IC engines using cyclostationarity. Part I: a methodology for condition monitoring, Journal of Sound and Vibration 257 (5) (2002) 815-837.

[4] J. Antoni, J. Danière, F. Guillet, R.B. Randall, Effective vibration analysis of IC engines using cyclostationarity. Part II: new results on the reconstruction of the cylinder pressure, Journal of Sound and Vibration 257 (5) (2002) 839-856.

[5] B. Lafon, J. Antoni, Cyclic sound intensity for noise separation diesel powertrain unit radiations, Proceedings of InterNoise 07, Istanbul, Turquey, 2007.

[6] B. Lafon, Noise Source Separation Coupled to Acoustical Imaging Techniques, Application to Sound Radiation of Diesel Engines, PhD Thesis (in French), University of Technology of Compiegne, France, 2009.

[7] B. Lafon, J. Antoni, M. Sidahmed, L. Polac, Cyclic sound intensity and source separation from NAH measurements on a diesel engine, Proceedings of Acoustics' 08, Paris, France, 2008.

[8] J. Antoni, F. Bonnardot, A. Raad, M. El Badaoui, Cyclostationary modelling of rotating machine vibration signals, Mechanical Systems and Signal Processing 18 (6) (2004) 1285-1314.

[9] J. Antoni, R.B. Randall, On the use of the cyclic power spectrum in rolling element bearing diagnostics, Journal of Sound and Vibration 281 (1-2) (2005) $463-468$

[10] J. Antoni, Cyclic spectral analysis of rolling-element bearing signals: facts and fictions, Journal of Sound and Vibration 304 (3-5) (2007) 497-529.

[11] A. Raad, J. Antoni, M. Sidahmed, Indicators of cyclostationarity: theory and application to gear fault monitoring, Mechanical Systems and Signal Processing 22 (3) (2008) 574-587.

[12] J. Antoni, Cyclostationarity by examples, Mechanical Systems and Signal Processing 23 (4) (2009) 987-1036.

[13] W.A. Gardner, Cyclostationarity in Communications and Signal Processing, IEEE Press, 1994,, pp. 168-239, 295-328.

[14] Q. Wan, W.K. Jiang, Near field acoustic holography (NAH) theory for cyclostationary sound field and its application, Journal of Sound and Vibration 290 (3-5) (2006) 956-967.

[15] H.B. Zhang, W.K. Jiang, Q. Wan, C.J. Liao, The application of wave superposition algorithm to identify cyclostationary sound source, Acta Acustica united with Acustica 94 (5) (2008) 649-655.

[16] M. El Badaoui, J. Danière, F. Guillet, C. Servière, Separation of combustion noise and piston slap in diesel engine by cyclic Wiener filtering, Mechanical Systems and Signal Processing 19 (6) (2005) 1209-1217.

[17] J. Antoni, R. Boustany, F. Gautier, S. Wang, Source separation in diesel engines with the cyclic Wiener filter, Proceedings of EuroNoise 2006, Tampere, Finland, 2006.

[18] L. Pruvost, Q. Leclère, E. Parizet, Diesel engine combustion and mechanical noise separation using an improved spectrofilter, Mechanical Systems and Signal Processing 23 (7) (2009) 2072-2087.

[19] F.J. Fahy, Sound Intensity, Elsevier Applied Science, London, 1989.

[20] J.S. Bendat, A.G. Piersol, Random Data: Analysis and Measurement Procedures, fourth ed., Wiley, 2010.

[21] R. Steiner, J. Hald, Near-field acoustical holography without the errors and limitations caused by the use of spatial DFT, The International Journal of Acoustics and Vibration 6 (2001) 83-89.

[22] J. Hald, Basic theory and properties of statistically optimized near-field acoustical holography, Journal of the Acoustical Society of America 125 (4) (2009) 2105-2120.

[23] S. Haykin, Adaptative Filter Theory, fourth ed., Prentice-Hall, 2001.

[24] Q. Leclère, L. Pruvost, E. Parizet, Angular and temporal determinism of rotating machine signals: the diesel engine case, Mechanical Systems and Signal Processing 4 (7) (2010) 2012-2020. 